\title{
A Systematic Approach to Restore Grossly Decayed Multirooted Teeth: Split Cast Post and Core
}

\author{
Lakshya Kumar, Ridhima Gupta, Akanksha Yadav
}

\begin{abstract}
Endodontically treated molars with almost total coronal destruction may be salvageable by using multiple posts in divergent canals. The fabrication of dowel and core for multirooted teeth can be a vexing problem, especially when there is minimal coronal dentin. A number of methods have been suggested for making a cast dowel and core using two or more of the canals to increase retention of the prosthesis. This article presents a clinical report on fabrication of split cast post and core by a directtechnique that is efficient and enables the dentist to use the maximum length available in each canal, despite the degree of divergence in multirooted teeth.
\end{abstract}

Keywords: Divergent root canal, Direct technique, Multirooted teeth, Split cast post and core.

How to cite this article: Kumar L, Gupta R, Yadav A. A Systematic Approach to Restore G rossly Decayed Multirooted Teeth: Split Cast Post and Core. Int J Prosthodont Restor Dent 2012;2(1):16-18.

\section{Source of support $\mathrm{Nil}$}

\section{Conflict of interest: None}

\section{INTRODUCTION}

Restoring grossly decayed multirooted teeth has al ways been challenging for any dentist and the laboratory technicians. Endodontist has to ensure the clinical success of endodontic procedures and the prosthodontist has to ensure the longterm durability and esthetics of the restoration. Cast post and cores provide retention and resistance to the already weakened tooth structure.

Rebuilding abutment teeth with the metal casting has been widely applied for a long time and has a good reputation clinically for grossly decayed teeth. This method brings difficulties when applied for the divergent and multirooted teeth. The split method has been used for the divergent and multirooted teeth, since it is difficult to insert the one piece casting dowel-core into the nonparallel root canals.

The fitting of cast post and core restorations is critical to ensure good adaptation and passivity of fit. ${ }^{1-3}$ If a passive fit is not achieved, wedging stresses may result in root fracture. ${ }^{4}$ In the case of multiple posts, parallelism of posts are required for seating of the restoration; but in cases where the roots are divergent split post and core are fabricated to achieve proper seating of the restoration. This article presents split cast post and core technique to restore grossly decayed mandibular second molar abutment for fixed partial denture.

\section{CASE REPORT}

A 25-year-old male patient reported with complaint of a missing left back tooth. On clinical examination revealed missing left mandibular first molar, grossly decayed mandibular left second molar and carious mandibular third molars. Radiographic examination revealed normal gingival and periodontal status.

\section{Treatment Plan}

A thorough clinical evaluation of the occlusion was done. A lginate impressions (Zelgan 2002, Dentsply, India) were made for making the diagnostic casts. The treatment plan included complete oral prophylaxis and maintenance protocol, root canal treatment of the left mandibular second molar followed by fabrication of split cast post and core and restoration of the carious teeth.

\section{Procedure}

After 3-month follow-up of endodontic treatment, guttapercha was removed from the pulp chamber and canals, leaving $4 \mathrm{~mm}$ in the apical portion of the distal canal. The canal was shaped with Peeso reamers (Dentsply, India). W eakened and unsupported tooth structure was removed. The custom-made acrylic resin post was inserted into the distal canal up to the calculated length. It should snugly fit into the canal. R oot canal surface was coated with a suitable lubricant. A n autopolymerized resin was applied on the custom-made acrylic post using brush bead technique and replaced into the distal canal. W hen the material is in dough stage, slowly withdraw the post and then again seat it into the place to prevent binding of acrylic resin into the root canal. A uxiliary post was fabricated using the same technique.

The primary post was lubricated with petroleum jelly. With the mesial post in place, Duralay resin was used to build up the core. Once the material is set primary post (distal canal) was removed first and then the auxiliary post (mesial canal) with core (Fig. 1). A high heat investment (B iovest, Dentsply International, Y ork, PA) that flows easily was used for casting. A uxiliary dow el with core was fitted in the tooth and split post (distal canal) was secured in its channel (Fig. 2). Radiograph of the post and core restoration was taken to confirm its proper seating (Fig. 3). 


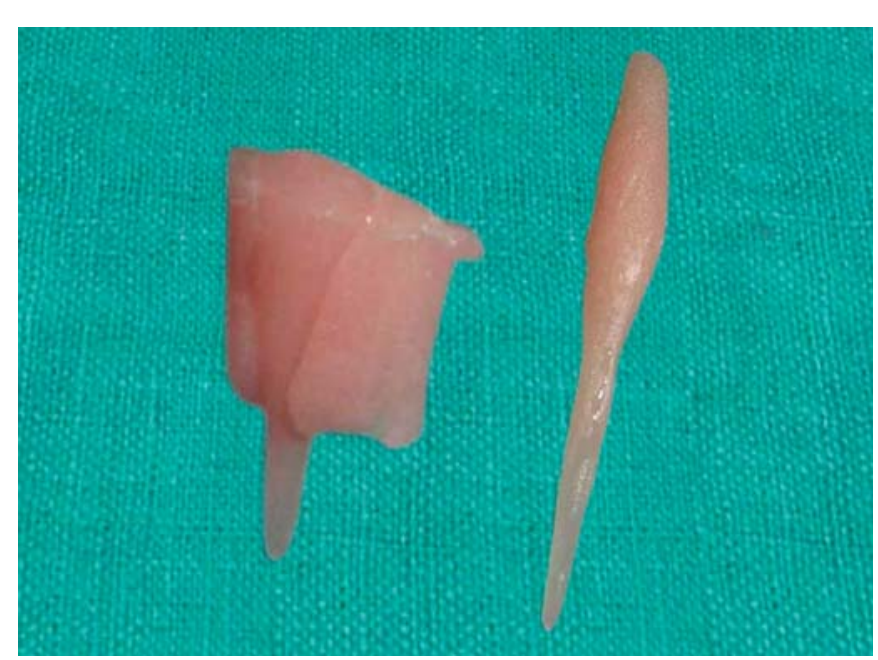

Fig. 1: Acrylic auxiliary post with core and primary post

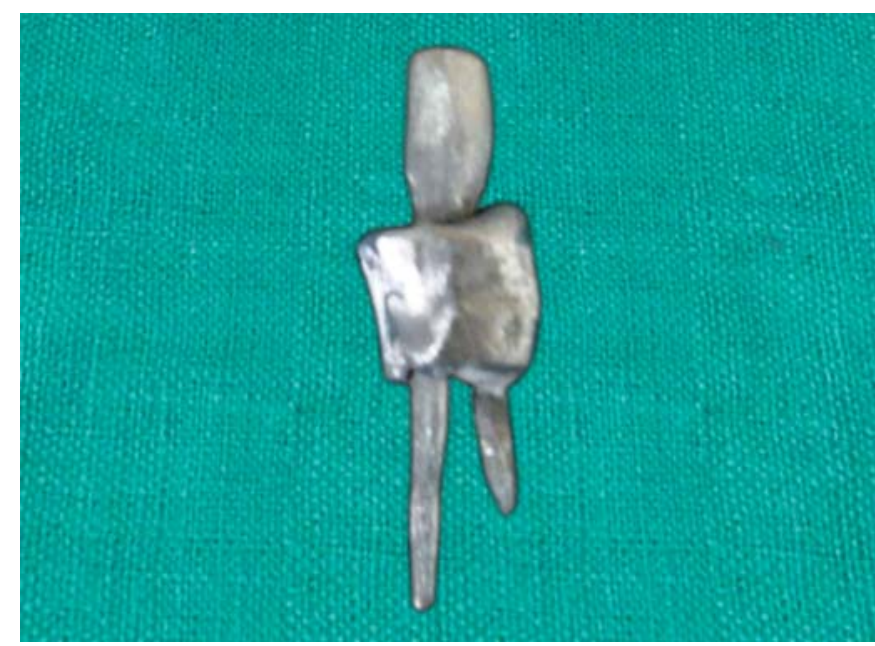

Fig. 2: Auxiliary post with core and primary post after casting

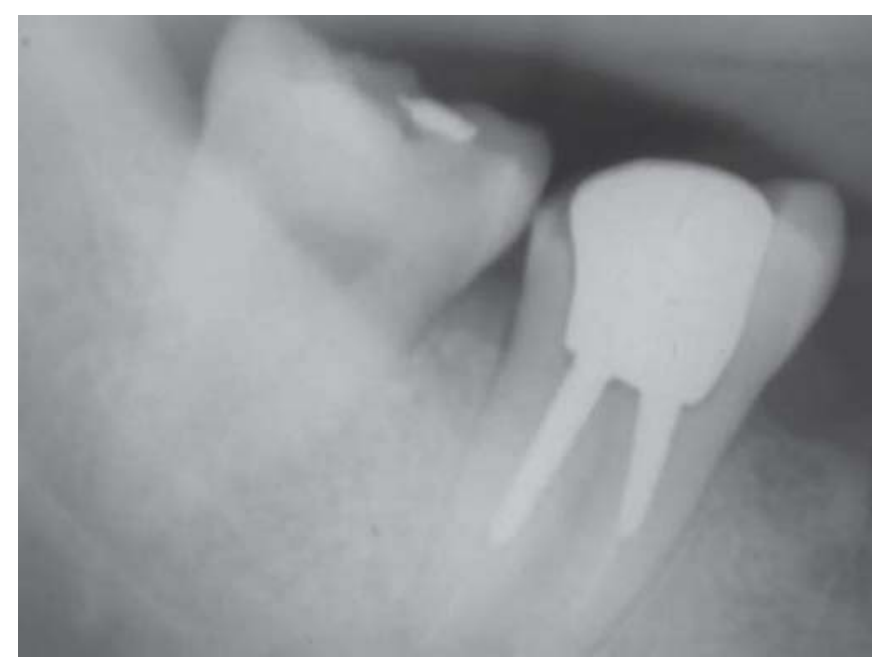

Fig. 3: Radiograph view of restoration

Tooth was cleaned and dried. First, the auxiliary dowel and core was cemented, and then the primary dowel. A fter the cement has set, primary dowel was cut flushing with

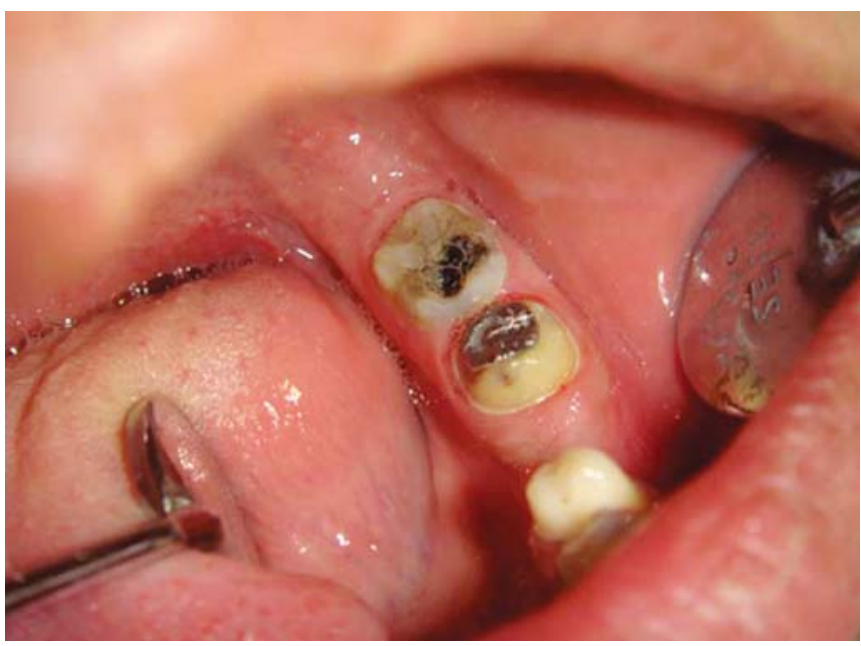

Fig. 4: Dowel flushing with the occlusal surface

the occlusal surface (Fig. 4). Two impressions were made; an alginate impression to prepare a temporary crown and another with hydrophilic polyvinyl siloxane impression material (dual mix) to prepare the metal-ceramic crown.

\section{DISCUSSION}

A post-and-core assembly is placed in a badly broken-dow $n$ tooth to restore the bulk of the coronal portion of the tooth to facilitate the subsequent restoration of the tooth by means of an indirect extracoronal restoration. Hitherto, dental cement, dental amalgam, metal casting and the combined method with the ready-made post and composite resin have been applied generally for the rebuilding of abutment teeth.

The literature describes numerous techniques for fabricating the cast post and core. ${ }^{5}$ Failure of these systems include loss of retention of posts and fracture of the root or root perforation. The present technique described in the article is effective for extensively damaged teeth that lack sufficient tooth structure to create an adequate ferrule of 1.5 to $2 \mathrm{~mm}$ for the final crown. The ultimate success of cast post and core depends largely on the level of education and motivation that the patient has gone through. At the 1-year clinical follow-up, the prosthesis exhibited no evidence of failure and the patient was satisfied with its function and esthetic.

\section{CONCLUSION}

The fabrication of dowels and cores for multirooted teeth can be a vexing problem, especially when there is minimal coronal dentin. This article describes a direct technique that is efficient and enables the dentist to use the maximum length available in each canal, despite the degree of divergence of the canals in multirooted teeth. 


\section{REFERENCES}

1. M organo SM, Brackett SE. Foundation restorations in fixed prosthodontics: Current knowledge and future needs. J Prosthet Dent 1999;82:643-57.

2. Goerig $A C, M$ ueninghoff $L A$. M anagement of the endodontically treated tooth. Part II: Technique. J Prosthet D ent 1983;49:491-97.

3. Sorensen J A, Engel man M J. Effect of post adaptation on fracture resistance of endodontically treated teeth. J Prosthet Dent 1990;64:419-24.

4. M organo SM, M ilot P. Clinical success of cast metal posts and cores. J Prosthet Dent 1993;70:11-16.

5. Sadan A, Elliot R, Raigrodski AJ. Treatment planning extensively broken-down mandibular molars for post-and-core fabrication. Q uintessence Int 1998;29(6):351-55.

\section{ABOUT THE AUTHORS}

\section{Lakshya Kumar}

Senior Lecturer, Department of Prosthodontics, Faculty of Dental Sciences, CSM M edical University (U pgraded KGMC), L ucknow Uttar Pradesh, India

\section{Ridhima Gupta (Corresponding Author)}

Senior Lecturer, Department of Prosthodontics, Institute of Dental Sciences and Technology, M eerut, U ttar Pradesh, India, Phone: +919560780215, e-mail: ridhim@gmail.com

\section{Akanksha Yadav}

Postgraduate Student, Department of Oral M edicine and Radiology UP Dental College, Lucknow, U ttar Pradesh, India 